\title{
Experiments and Evaluation of Public Policies: Methods, Implementation and Challenges $^{1}$
}

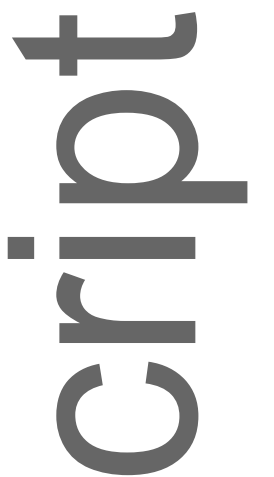

\author{
Professor Paul H. Jensen \\ Faculty of Business and Economics \\ University of Melbourne VIC 3010 \\ Email: pjensen@unimelb.edu.au
}

\begin{abstract}
Evidence-based policymaking is all about developing and implementing better public policies. Although the logic underpinning this philosophy is simple, the practicalities of demonstrating causal effects of a public policy are much more complex. In recent years, there has been a wave of optimism about the usefulness of experimental approaches to public policy evaluation which mimics the clean, causal inferences observed in clinical trials. Although these methods, such as randomised controlled trials (RCTs), have been widely advocated and implemented, they are not without their potential problems. In this paper, we consider the strengths, weaknesses and challenges posed by the revolution in policy evaluation brought about by embracing experimental methods.
\end{abstract}

\section{Introduction}

Evidence-based policymaking is all about developing and implementing better public policies, thereby creating healthier and wealthier societies, through deductive logic and statistical inference. The rigour underpinning evidence-based policies should provide taxpayers with more confidence that the Government is spending prudently - that the programs the Government invest in produce desirable economic and social returns. Although there are many ways in which robust evidence can be created and implemented, one increasingly-important approach adopts an experimental approach to public policy

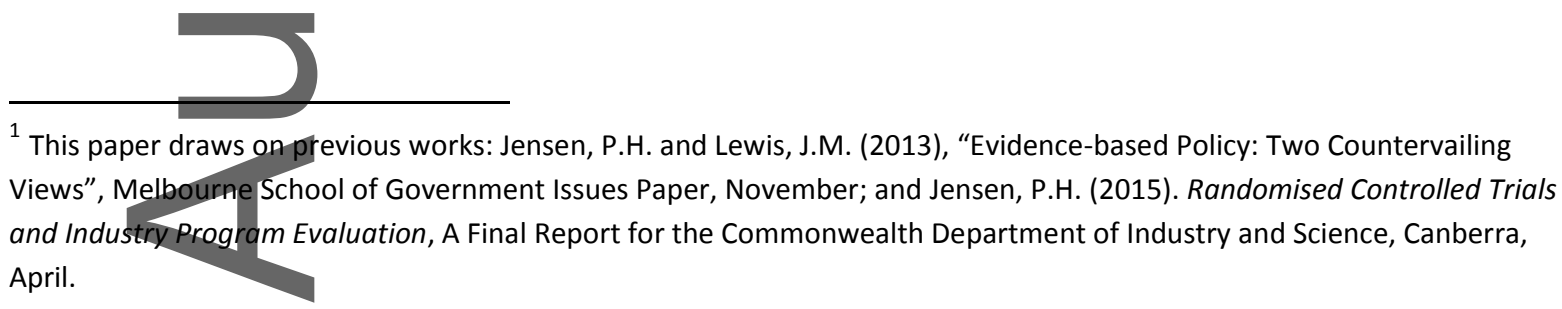

This is the author manuscript accepted for publication and has undergone full peer review but has not been through the copyediting, typesetting, pagination and proofreading process, which may lead to differences between this version and the Version of Record. Please cite this article as doi:

$\underline{10.1111 / 1467-8500.12406 .}$.

This article is protected by copyright. All rights reserved. 
which mimics the approach taken in medicine and science more generally with regard to determining the effectiveness of a specific government intervention.

This paper analyses the potential for experimental methods and randomised controlled trials (RCTS) to be used to evaluate a range of public policy initiatives and programs. This will involve a review of the extant literature on the rationale, methods and outcomes of RCTs around the world. Although many of the applications considered in the literature are focused on economic development and poverty alleviation issues, there is an emerging literature on different policy domains - e.g. industry policy, health policy, education policy which will also be considered. Despite their widespread use and acceptance - partly due to their clear and simple logic - there has also been a surge in criticisms around the use of RCTs which has challenged their legitimacy.

\section{Background}

The logic of evidence-based policy is simple and compelling: since the world is complex, it is not straightforward to determine whether a policy has worked. Therefore, we need to invest effort into evaluating which policies 'work' in the sense that they produced the desired outcomes. The use of evidence to evaluate public policy programs - rather than relying on ideology, vignettes, assertions or opinions - should ensure that good policies survive and bad policies are killed off (assuming, of course, that we all agree on the outcomes that a policy is supposed to achieve and how they can be accurately measured, both of which are non-trivial matters). A key challenge in evaluation is determining and isolating 'what works' since most programs are instituted in complex settings where there are many factors that influence their success (e.g. the effects of 'good programs' could be overwhelmed by factors such as a global economic downturn). To help us achieve this goal, we need a set of tools and techniques to help guide us. If you accept the logic outlined above, the key challenge is to identify a counterfactual: what would have happened in the absence of participating in the program? This focus on causality (including the direction of causality) is at the heart of the evidence-based policy framework (see Productivity Commission 2009 for an overview of the issues). 
Banks (2009) argues that there are certain hallmarks of all good evaluations - for example, they test a proposition as to why a specific policy might work; they consider a counterfactual and attempt to quantify the size of an effect which includes both direct and indirect effects; include sensitivity tests to ensure that the estimated effects are robust; and can be tested and replicated by third parties. In Australia, according to Banks (2009), there are many Australian examples of good policy reform that have been guided and argued by evidence-based policy. This includes the reduction of tariffs on imports which occurred in the 1970s, the Higher Education Contribution Scheme (HECS) which was designed by ANU Professor of Economics, Bruce Chapman (based on an idea espoused by Milton Friedman (1955)), lifetime community rating on private health insurance, and national competition policy.

Focusing on the non-political objectives of a program, it seems obvious that all policymakers want the best possible outcomes given the prevailing economic and social conditions. However, programs have both intended and unintended consequences - as well as both direct and indirect effects - which need to be considered. For example, programs often have effects that ripple throughout the economy, not just on the target group of interest. For example, a policy designed to impact female labour participation might directly affect mothers decisions about if (and when) to return to work (casual, part-time or full-time), but are also likely to have indirect effects on the demand for childcare services, take-away meals and dry-cleaning services. An evaluation of this program which adopted a macro perspective would capture all these effects (the positive effect of increased labour productivity and the negative effects associated with reduced aggregate demand) in order to determine whether the policy was good for society or not. Since this is complex, evaluations often resort to the simpler (but still difficult) task of evaluating the effects of the program on the target group: that is, whether the program has succeeded in increasing female workforce participation.

Program evaluation must be undertaken carefully. The consumers of this analysis are ultimately the central agencies who control the purse strings of the government departments: 'high-quality' evidence is needed to convince them of the merits of the program. There are a range of methods and techniques that can be used to examine the effects of specific programs. These methods produce the 'evidence base' from which to build better policy. But because not all evidence produced by these methods is the same 
quality, it is equally important to be able to rank them according to the quality of evidence produced. That is, to provide a hierarchy of evidence - in which the gold standard will provide robust causal evidence of the effects of a specific program. Of course, this is a highly-contested debate and there is even the most ardent of evidence-based advocates will admit that causality is impossible to establish definitively.

It is common to confuse monitoring with evaluation. Whereas monitoring fulfils an auditing function - reporting on how the moneys were spent, the number of people participating in a program and the numbers still in business or employment - an evaluation aims to establish what would have happened to the people or the business in the absence of the program. Competent monitoring is a necessary condition for probity and efficient policy but it does not tell us whether public funds have been spent in a manner that delivers the maximum intended benefits to citizens; hence the need for evaluation.

Microeconomic evaluations use a control group to separate the effects of the program from other confounding influences - e.g. the fact that businesses choose to participate in a program (participation is not random). If the best or most persistent and determined companies select themselves into a program, then it is very difficult to disentangle the effects of the program from the effects of persistence and determination. Experimental approaches have become increasingly popular since the 1990s (see Meyer 1995). Although they are not the only way to estimate a counterfactual; if designed and executed well, they represent an extremely clean estimate of the effects of a program intervention.

\section{Program evaluation and natural experiments}

Before embarking on a discussion of the strengths and limitations of using RCTs for program evaluation, it is necessary to outline some basics about the types (and objectives) of evaluation questions. In general, there are three distinct program evaluation questions:

1. What is the effect of the program on participants and non-participants compared to noprogram at all?

2. What is the effect if the program were to be applied to a new environment? (Heckman 2000); and 
3. What is the opportunity cost of the program - that is, what are the benefits foregone from either i) not lowering taxes or ii) spending on another program?

These questions require different evaluation methods. To address the first question, the common evaluation method is based on estimation of a 'treatment effect'. The underlying idea of the treatment effect approach is to mimic the hard science approach: the average outcome of exposure to the policy (the treatment) is compared to the average outcome of others (the control). The second question is harder than the first: it requires answers based on estimates that are of higher degree of interpretability, transportability and comparability than the ones produced by the treatment effect approach. In other words, to answer the second question requires estimation of tightly specified economic structural models (Heckman 2000).

The third question is more difficult again because it requires defining a reasonable alternative use of funds. This requires addressing the fact that policies have effects that ripple throughout the economy, not just on the target group of interest. In this sense, the determination of whether a program 'works' captures all of the economic consequences of participation in the program. For example, a program designed to impact the uptake of solar panels (e.g. via a Government rebate) will undoubtedly affect households' consumption of electricity off the grid which might reduce jobs in coal-mining regions (the net effect on jobs is unclear since jobs will be created in solar panels), as well as an effect on consumption of other goods/services since the household's total energy bill might now be smaller.

One of the most difficult issues in conducting a program evaluation is removing the effects of self-selection: i.e. the fact that businesses choose to participate in a program. This is essentially the problem of defining a suitable control group. If the best or most persistent and determined companies self-select into a program, then it is very difficult to disentangle the effects of the program from the effects of persistence and determination. The best way to deal with this is to construct a control group of firms from the population that is similar (using observable characteristics such as size, location, industry etc.) to the treatment group of firms but for some reason has not participated in the program. This is often done with techniques like propensity score matching, which systematically finds 'nearest neighbours' to the control group using a set of observable characteristics. However, this approach is not 
always possible. In these instances, it may be appropriate to construct a control group ex post and note the direction of the bias due to unmeasured confounding factors.

The performance of the treatment and control groups can then be evaluated using a range of different techniques, which may involve estimating specific parameters of interest or simpler approaches such as difference-in-differences. With the advent of more (and cheaper) data, econometricians believe that the self-selection problems can be effectively handled within their approach either by measuring more previously-unmeasured characteristics or by use of instrumental variables. Despite this, econometricians seem to be more concerned than ever about self-selection on unobservable characteristics, particularly those that are psychological in nature and therefore extremely difficult to observe (see Ravallion 2012).

In addition to these approaches which use observational data, there are two experimental approaches to constructing a counterfactual: natural experiments and RCTs. Natural experiments rely on statistical variation induced by random forces: in this approach, a treatment and control group are naturally constructed when a treatment exogenously occurs to one part of a population and not another. By 'exogenous' we mean the affected people had no choice in whether they participated or were affected by the event. For example, an exogenous shock might be a natural disaster (e.g. a cyclone) that affects one banana-producing region in Queensland but not another banana-producing region in NSW. Because supply and demand are determined simultaneously, it is a non-trivial problem in economics to determine whether observed changes in prices or quantities are due to demand-side factors or supply-side factors. So, economists use exogenous shocks like the cyclones in Queensland in 2006 and 2011 (which only affect the supply-side) to examine important policy jssues like the effect of import restrictions on prices (see Ko, Frijters and Foster 2018). The main problem with this method is that pure, natural experiments occur by chance and cannot be produced on demand.

Other natural experiments exploit random assignment of people (or firms) to 'treatment' and 'control' groups in order to analyse the effects of a specific public policy. Consider an example where we want to understand the effect of participation in the armed forces on future earnings. This would be impossible to do if we simply compared the earnings performance of the two types of workers in a specific industry (e.g. mechanics) - those who 
had previously served in the Army and those who hadn't. The reason you couldn't do the simple comparison is that there may be non-random factors which determine who decides to join the Army in the first place, which means that the two groups aren't comparable. This problem can be addressed in certain situations. For example, Australia introduced a lottery system of conscription to the armed forces during the Vietnam War (e.g. see Siminski 2013). Under this system, people were randomly forced to join to army depending on whether their number was called in the lottery (of course, some still refused to join even if their number was called, which suggests there may still be some selection bias). Another interesting Australia example of the use of natural experiments relates to the baby bonus, which was first introduced in 2004. Some economists use this policy shock (no-one knew it was going to beintroduced) to examine the effect of family income on student outcomes at Grade 3 by comparing those children whose family received the $\$ 3,000$ bonus with those families who didn't (i.e. were born prior to the introduction of the baby bonus scheme). It appears this did have some effect for children from disadvantaged backgrounds (Deutscher and Breunig 2017).

Randomised controlled trials (RCTs) are an example of a type of experimental method: one in which the experiment isn't 'natural' but is rather 'designed'. Due to their preoccupation with randomisation, researchers using this method have been referred to as 'randomistas'. Recent literature has considered how researchers using these methods have transformed the way we think about policy interventions (Leigh 2018). In other words, firms or governments deliberately design an experiment to examine the effect of a specific policy change. As long as these firms are drawn from the same population - and we can observe a large number of firms before and after the program - then we can be confident that any unobserved differences (e.g. the skill of senior management) in firm performance-related characteristics will be evenly allocated across the groups. This means that confounding effects from unmeasured factors are accounted for. This is the cleanest way to overcome the self-selection problem discussed above (without random assignment, people may simply choose to be in the treatment or control group based on their preferences).

Despite their benefits, there are still concerns about the usefulness of RCTs in social science contexts. Heckman and Smith (1995) suggest that these shortcomings are acute. One obvious pitfall in the social sciences is the lack of a 'placebo': in medical trials, two groups 
are given pills, but one is given a pill which turns out to have no active ingredient. This approach simply can't be imitated in the social sciences: the people in the control group know that they aren't receiving the treatment (it is impossible to fool them into thinking they might be receiving a treatment when they aren't).

The main strengths and weaknesses are considered here. One such concern relates to ethical issues: some people argue that it is unethical to simply toss a coin to determine who receives the 'treatment'. RCT proponents counter that it is only unethical to conduct the trial if we already know that the program works. If you don't know whether a specific policy works, it is unethical i) to do nothing; or ii) not to conduct an experiment to find out what works. However, there is concern amongst some development economists that experiments have been used in areas where we do know whether the program works: for example, medical treatments (see Ravallion 2012). On top of this, there are issue about informed consent' since some evaluations are conducted in developing country villages where they are not asked whether they would like to be part of an experiment.

Moreover, RCTs can't be used in all contexts. For example, it is impossible to design and conduct an experiment on macroeconomic issues such as a random shock to interest rates. How can we assume country comparability and how could this be applied to one country but not to another? In addition, it has been argued that a fascination with experiments may lead researchers to avoid important policy issues that can't be solved using experiments (see Deaton 2010). For example, Angrist and Pischke (2010) state that "critics of designdriven studies argue that in pursuit of clean and credible research designs, researchers seek good answers instead of good questions" (p.6).

What may be a more serious concern relates to what is known as 'generalisability', which is simply the problem that results observed in one experiment might not hold in other contexts. RCTs are typically conducted in environments with unique characteristics which may not be representative of all possible environments. Therefore, the results observed in one setting might not be generalizable to all contexts. Problems of this nature arise in nonexperimental analysis too. But, according to Glennerster (2013), experiments tend to get criticised for this shortcoming more than other methodological approaches simply because experiments have solved most of the other methodological issues. 
The whole premise of the RCT approach is that treatment and control groups are similar but distinct. However, this may not be correct as there is the potential for the treatment and control groups to be contaminated either i) because of information flows from treatment to the controlgroup; or ii) because the act of receiving a treatment changes the very nature of the competition between agents in the treatment and control groups. Although it is not always possible to solve these problems perfectly, it is possible to geographically separate participants in treatment and control groups so that information is harder to transmit (although this is imperfect given the cost of communication nowadays) and to increase the sample size of the groups so that the estimate of the treatment effect converges on the true treatment effect.

One of the staunchest critics of the new wave of interest in randomisation is Angus Deaton, winner of the Nobel Memorial Prize in Economic Sciences in 2015. On a very technical note, he points out that there are two stages to the process of determining the 'treatment' and 'control' groups. Jake a population of units from which you want to draw the two groups. The first stage is to select a 'treatment panel': those individual units which are willing to be part of the experiment. The second stage involves randomly allocating each of the units in the treatment panel to the 'treatment' or the 'control' group. One of the virtues put forward by advocates of experiments relates to the fact that they are free of (self-) selection bias. But this is only true with regard to the second stage of the process noted above: in the first stage, it is necessary to select which units in the population will participate in the experiment and this might not be done randomly (see Deaton 2012).

Also note that experiments provide a 'mean' effect (not a 'median' effect, and not a percentage of people whose position improved). So, just because a policy produces a positive effect on average doesn't mean that everyone participating in the program will experience the average effect. Of course, there is a distribution around the average: and if the distribution is spread widely (i.e. there is a high variance), the performance of a given individual could be much better (or much worse) than the average. However, as Imbens (2010) points out (following Manski 1996), a social planner could always compare the average effects with/without treatment and the change in the dispersion of the effect with/without treatment. 
While RCTs tend to be held as the most 'rigorous' (i.e. how certain we are that the estimated program impact is accurate), they can be expensive to operate, difficult to negotiate and take a long time to undertake. While an RCT may give an impact estimate that is $99 \%$ certain, a difference-in-difference estimate may be $80 \%$ certain. In some cases, the latter is all that is required for good policy. In the debate over the rank-ordering of different evaluation methodologies, Imbens makes the following point: "I do not want to say that, in practice, randomized experiments are generally perfect or that their implementation cannot be improved, but I do want to make the claim that giving up control over the assignment process is unlikely to improve matters" (Imbens 2010, p.412). In other words, it is hard to mount a convincing case that giving up randomisation will unambiguously improve the state of policy evaluation practice. So, if randomisation is possible, it should be pursued. However, it may be the case that an RCT is not the most cost-effective way to proceed. In addition, RCTs might be difficult to implement in some contexts.

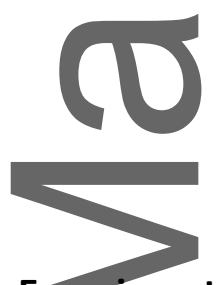

\section{Experimental design methods}

The classic and simplest way of introducing randomisation into an evaluation is to do so at the initiation of a program as is done in clinical trials. This ensures that the process is likely to produce the most reliable results possible. However, this is not always possible because the frontier in the application of RCTs evaluation methods is in social settings rather than laboratories. There are a range of alternative approaches that have been developed primarily by people working in development economics - with regard to introducing randomisation into both new and existing programs: including over-subscription, phase-in, within-group randomisation and encouragement design (see Duflo, Glennerster and Kremer 2007). In this section, we cover the basic approaches to conducting RCTs and consider some of the alternative methods that are used to introduce randomisation into the evaluation process.

Simple ' 1 treatment' approach. This is the simplest approach where there is simply 1 treatment and 1 control group. 
Multiple treatments. It is possible to introduce multiple treatment groups into the analysis - and then the performance of the treatment groups can be compared or that treatment group(s) can be compared against a control group. One interesting approach is to have three different groups: one that receives Treatment \#1, one that receives Treatment \#2 and a third group that receives both Treatments \#1 and \#2. A control group receives none of the treatments. So, this approach provides a great deal of flexibility with regard to the evaluation of the effectiveness of different programs.

Over-subscription. In some instances where it isn't possible to introduce randomisation at the start of a program - or where it is not politically palatable to do so - it may be possible to introduce randomisation (in the form of a lottery) for programs where demand outstrips supply. In other words, if there is only a finite number of slots available in a program (due to the scarcity of funds), then it is possible to allocate some (or all) of the slots on a lottery basis.

Phase-in. There are situations where practical or financial constraints may mean that it is simply not possible to implement a new program all at once, so it needs to be phased in over time.-Determining who should be phased in at different stages of the program can be done via randomisation. This can be an effective approach to randomisation, but it does suffer from some drawbacks. For example, it won't work in instances where there is likely to be contamination between the groups in different phases such as might occur when people modify their behaviour in Phase 1 because of the expectation of being part of the program in Phase 2. In that case, participants in Phase 2 are not an appropriate group for comparison in Phase 1.

Within-group randomisation. It is not always possible to provide services to one group and not to another group, as is required in the clinical trial approach. In this case, it is common to use within-group randomisation which effectively means that different members of a group will receive the treatment. For example, in a school situation where a new program is intended to be implemented, it could be that students in Grade 3 at School A will receive the treatment whereas students in Grade 4 at School B will receive the treatment. On equity grounds, it is deemed acceptable that Grade 3 students at School B don't receive the treatment as long as students in Grade 4 do. That is, what matters is that all of the schools receive equal treatment.

This article is protected by copyright. All rights reserved. 
Randomising within a bubble. In situations where scoring is used to evaluate proposals on a merit basis, this approach is particularly useful. It relies on the fact that 'merit' is often difficult to perfectly measure and so many of the proposals that are just under the merit threshold score could actually be 'potentially fundable' (which is sometimes referred to as a "bubble"). Take an example where the merit score threshold is 70: if there is statistical noise (i.e. error) in the way the scores are determined, it is entirely possible that an application with a score of 69 is equivalent in quality to another which scores 70 . Yet, only the application with a score of 70 is funded. By randomly providing funds to some of these applications that scored just below the threshold in the treatment group, you get a richer set of comparisons since you can consider the treatment group, the potentially fundable group plus a reference group (that is not funded).

Weighted randomisation. One of the common concerns about a pure randomisation approach to allocating resources is that it doesn't take into account the quality of the applicants. This is particularly a concern in merit-based programs where the basic idea is to allocate monies to people/firms with the best ideas/projects. Of course, there is always statistical noise in the way we evaluate the merits of different proposals (i.e. there are measurement issues which mean we don't know the 'true' underlying quality), but nevertheless merit-based programs typically involve making serious investments into scoring applications. The main benefit of the weighted randomisation approach is that it puts a 'weight' on higher-ranked proposals, thereby increasing the likelihood that they will be granted some money (which satisfies some equity considerations). And noise can potentially be reduced by the introduction of stratified random assignment, which can identify homogeneous sub-groups within a population.

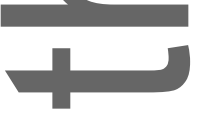

\section{Conclusions}

Evidence-based policymaking is all about developing and implementing better public policies, thereby creating healthier and wealthier societies, through deductive logic and statistical inference. Although there are many ways in which robust evidence can be created and implemented, one increasingly-important approach adopts an experimental approach to public policy which mimics the approach taken in medicine and science more generally with regard to determining the effectiveness of a specific government intervention. These 
experimental approaches have been very popular amongst economists who believe they provide more credibility to the causal narrative underpinning evidence-based policy. However, they have also been subject to substantial criticisms from those who believe their benefits have been overstated relative to other methods.

In this paper, we take a careful and thorough look at the usefulness of experimental methods and randomised controlled trials (RCTs) in evaluating the performance of government policy programs. The broad conclusion from this discussion is that there is enormous potential to introduce more rigorous evaluation of government programs in a range of policy domains and RCTs should play an important role in this. However, RCTs are certainly no policy panacea - the discussion here has illustrated the fact that there are many challenges associated with the design, implementation and interpretation of findings based on RCTs - but they nevertheless provide a conceptually clear and methodologically sound way of beginning to figure out what works (and what doesn't) in a complex policy environment.

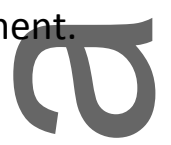

\section{Acknowledgements}

This paper draws on previous works: Jensen, P.H. and Lewis, J.M. (2013), “Evidence-based Policy: Two Countervailing Views", Melbourne School of Government Issues Paper, November; and Jensen, P.H. (2015). Randomised Controlled Trials and Industry Program Evaluation, A Final Report for the Commonwealth Department of Industry and Science, Canberra, Aprit

\section{References}

Angrist, J.D. and Pischke, J.-S. (2010). "The credibility revolution in empirical economics: How better research design is taking the con out of econometrics", Journal of Economic Perspectives 24(2): 3-30.

Deaton, A. (2012), "Searching for answers with randomized controlled trials", presentation at NYU Development Research Institute, March 22, 2012.

Deutscher, N. and Breunig, R. (2017), "Baby bonuses: Natural experiments in cash transfers, birth timing and child outcomes", Economic Record 94, 1-24.

Duflo, E., Glennerster, R., and Kremer, M. (2007). "Using randomization in development economics research: A toolkit", Handbook of Development Economics, 4, 3895-3962. 
Friedman, M. (1955). 'The role of government in education' in Capitalism and Freedom, Chicago University Press, Chicago.

Glennerster, R. (2013). Presentation at "Evidence-Based Policy-Making: Meeting the Challenges", $5^{\text {th }}$ July 2013, Canberra.

Heckman, . (2000). "Microdata, Heterogeneity and The Evaluation of Public Policy", Bank of Sweden Nobel Memorial Lecture in Economic Sciences, December 8, Stockholm, Sweden.

Heckman, J.J. and Smith, J.A. (1995). "Assessing the case for social experiments", Journal of Economic Perspectives 9(2): 85-100.

Imbens, G (2010). "Better LATE than never: Some comments on Deaton (2009) and Heckman and Urzua (2009)", Journal of Economic Literature 48 (June): 399-423. Jensen, P.H. (2015). Randomised Controlled Trials and Industry Program Evaluation, A Final Report for the Commonwealth Department of Industry and Science, Canberra, April.

Ko, C.C., Frijters, P. and Foster, G. (2018). "A tale of cyclones, exports and surplus foregone in Australia's protected banana industry", Economic Record 94, 276-300.

Leigh, A. (2018). Randomistas: How Radical Researchers Changed the World, La Trobe University Press.

Manski, C. (1996). "Learning about treatment effects from experiments with random assignment of treatments", Journal of Human Resources 31(4): 709-33.

Meyer, B.D. (1995). "Natural and quasi-experiments in economics", Journal of Business \& Economic Statistics 13(2), 151-161.

Productivity Commission (2009). Strengthening Evidence-Based Policy in the Australian Federation, Roundtable proceedings, Canberra, $17-18^{\text {th }}$ August, Volume 1.

Ravallion, M. (2012). "Fighting poverty one experiment at a time: A review of Abhijit Banerjee and Esther Duflo's Poor Economics: A Radical Rethinking of the Way to Fight Global Poverty", Journal of Economic Literature 50(1): 103-14.

Siminski, P. (2013). "Employment effects of army service and veterans' compensation: Evidence from the Australian Vietnam-Era Conscription Lotteries", Review of Economics and Statistics 95(1), 87-97.

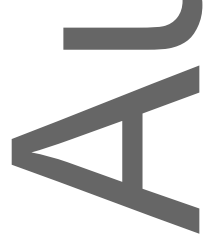




\section{University Library}

\section{- M M I N E R VA A gateway to Melbourne's research publications}

Minerva Access is the Institutional Repository of The University of Melbourne

Author/s:

Jensen, $\mathrm{PH}$

Title:

Experiments and evaluation of public policies: Methods, implementation, and challenges

Date:

2020-06-01

Citation:

Jensen, P. H. (2020). Experiments and evaluation of public policies: Methods, implementation, and challenges. Australian Journal of Public Administration, 79 (2), pp.259-268. https://doi.org/10.1111/1467-8500.12406.

Persistent Link:

http://hdl.handle.net/11343/276822 